\title{
Cell-based approach for 3D reconstruction of lymphatic capillaries in vitro reveals distinct functions of HGF and VEGF-C in lymphangiogenesis
}

Laure Gibot ${ }^{1}$, Todd Galbraith ${ }^{1}$, Bryan Kloos ${ }^{2}$, Suvendu Das ${ }^{2}$, Dan A. Lacroix ${ }^{1}$, François A. Auger $^{1^{*}}$, Mihaela Skobe ${ }^{2 * \dagger}$

${ }^{1}$ Centre LOEX de l'Université Laval, Regenerative Medicine section of the FRQS Research Center of the CHU de Québec, Quebec G1J1Z4, QC, Canada

${ }^{2}$ Department of Oncological Sciences and Tisch Cancer Center, Icahn School of Medicine at Mount Sinai, New York, NY 10029, USA

*These authors contributed equally to this work

${ }^{\dagger}$ Correspondence should be addressed to Mihaela Skobe (mihaela.skobe@mssm.edu)

Short title: In vitro human lymphatic microvascular network

Abbreviations: LEC: lymphatic endothelial cell; HGF: hepatocyte growth factor; VEGFR-3: vascular endothelial growth factor receptor-3 


\begin{abstract}
Regeneration of lymphatic vessels is important for treatment of various disorders of lymphatic system and for restoration of lymphatic function after surgery. We have developed a method for generating a human 3D lymphatic vascular construct. In this system, human lymphatic endothelial cells, co-cultured with fibroblasts, spontaneously organized into a stable 3D lymphatic capillary network without the use of any exogenous factors. In vitro-generated lymphatic capillaries exhibited the major molecular and ultra-structural features of native, human lymphatic microvasculature: branches in the three dimensions, wide lumen, blind ends, overlapping borders, adherens and tight junctions, lack of mural cells, no basement membrane, and anchoring filaments. Furthermore, we show that fibroblast-derived VEGF-C and HGF cooperate in the formation of lymphatic vasculature by activating ERK1/2 signaling, and demonstrate distinct functions of HGF/c-Met and VEGF-C/VEGFR-3 in lymphangiogenesis. In conclusion, this lymphatic vascular construct is expected to facilitate studies of lymphangiogenesis in vitro and it holds promise as a strategy for regeneration of lymphatic vessels and treatment of lymphatic disorders in various conditions.
\end{abstract}

Key words: lymphangiogenesis, lymphatic capillary, HGF, c-Met, VEGF-C, VEGFR-3, 3D, self-assembly 


\section{INTRODUCTION}

The lymphatic vascular system plays a pivotal role in the regulation of tissue homeostasis. By returning fluids and macromolecules from the interstitium back into the blood circulation, lymphatics control interstitial fluid pressure and protein balance. Lymphatics also regulate retention and clearance of sodium stored in the skin and failure of this process results in skin electrolyte accumulation and arterial hypertension[1]. Lymphatics influence lipid metabolism and loss of lymphatic vessel integrity results in fat accumulation and ultimately, obesity[2, 3]. Lymphatic vessels have also emerged as important regulators of immunity and inflammation[4, 5]. Recent evidence shows that LECs directly control traffic of immune cells, promote peripheral tolerance and scavenge inflammatory mediators, in addition to the classic role of lymphatic vessels in transporting leukocytes and antigens from peripheral tissues into the lymph nodes. Hence, lymphatics are essential for proper function of many tissues, and dysfunction of lymphatics leads to a vast array of consequences, including lymphedema, fibrosis and persistent inflammation amongst others.

Therapeutic lymphangiogenesis is an important strategy for treatment of many conditions in which lymphatic vessels are damaged or their function impaired. Such conditions range from congenital lymphatic malformations to acquired lymphatic insufficiencies caused by injury, surgery, or radiation treatment[6]. Although there has been considerable interest in lymphangiogenesis in recent years, the advances in developing methods for lymphatic regeneration and repair have been modest and mechanisms of lymphangiogenesis are incompletely understood. Relevant in vitro models of the human lymphatic vasculature are essential for enabling such studies, yet models are scarce, and most of them do not adequately recapitulate features of native lymphatics.

Studies of lymphangiogenesis rely mainly on the use of 2D cell culture models and most 3D models are classic tube formation assays adapted from studies of angiogenesis in 
vitro[7]. Approaches commonly involve use of biological matrices such as Matrigel, fibrin and collagen[8-10]. Matrigel, which has been widely used[11], is not a native extracellular environment for LECs, since lymphatics have poorly developed basement membrane[12]. Collagen type I and fibrin are more relevant ECM for LECs, however, on all of these matrices LECs form only simple cord-like structures and not bona fide lymphatic vessels with lumen. Another disadvantage of these culture systems is that the formation of endothelial cords is only transient, and newly formed tubes rapidly regress, usually within $24-48 \mathrm{~h}$ period. The in vitro model which thus far most closely recapitulates process of lymphangiogenesis is the mouse thoracic duct model, an adaptation of the aortic ring angiogenesis assay[13]. This model, however, also has its limitations since it utilizes segments from large lymphatic vessels and not lymphatic capillary endothelium, and it cannot be adapted for human studies.

Few attempts have been made to employ tissue-engineering strategies to generate human lymphatic vasculature in vitro $[14,15]$. LECs have been cultured in polyglycolic acid (PGA) scaffolds and transplanted into mice, however, LECs in this system do not form genuine lymphatic vessels, but instead merely line the scaffold pre-formed into tubes[16]. To engineer skin grafts with blood and lymphatic capillaries, human microvascular dermal endothelial cells (HDMECs), which consist of a mixture of blood endothelial cells (BECs) and lymphatic endothelial cells (LECs), have been embedded in fibrin and collagen type I 3D hydrogels and shown to generate blood and lymphatic vascular network[17]. Upon grafting onto the animals, lymphatics within the skin graft anastomosed with the host vessels and improved fluid drainage from the wound. This important work demonstrated for the first time feasibility of generating lymphatic vasculature in a scaffold-based construct suitable for transplantation. Such tissue-engineering approaches for lymphatic vessel reconstruction hold great potential not only for treatment of lymphatic insufficiencies, but also for improving the 
function and organization of complex bioengineered tissues and organs by incorporating lymphatic vasculature.

Here, we used cell-based approach which does not require use of any biomaterials, to generate human 3D lymphatic microvascular network in vitro. We developed a simple system in which lymphatic endothelial cells co-cultured with fibroblasts spontaneously organize into a 3D lymphatic capillary network which remains stable over at least several weeks. Lymphatic vessel reconstruction was driven entirely by VEGF-C and HGF endogenously produced by fibroblasts. Lymphatic network was branched in the three dimensions and displayed lumens, blind ends, typical junctions and all other key structural and molecular characteristic of lymphatic capillaries[12]. This reproducible system allows assaying modulators of lymphangiogenesis, computer-assisted quantitative analyses and it is amenable for transplantation.

\section{MATERIALS AND METHODS}

Reagents. Recombinant human HGF and VEGF-A were purchased from Peprotech. rhVEGF$\mathrm{C}$ was expressed and purified as previously described[10]. The c-Met tyrosine kinase inhibitor SU11274 (Selleck chemicals) was used at a final concentration of $1 \mu \mathrm{M}$. The anti-hVEGFR-3 blocking antibody hF4-3C5 was obtained from ImClone Systems and used at final concentration of $2.5 \mu \mathrm{g} / \mathrm{ml}[18]$. For MAPK inhibition, LECs were pretreated with a mitogenactivated protein kinase kinase (MEK1/2) inhibitor, U0126 at $10 \mu \mathrm{mol} / \mathrm{L}$ (Cell Signaling) for 1h before being utilized in the appropriate assay.

Cell isolation and culture. Human lymphatic and blood endothelial cells (LECs and BECs, respectively) were isolated from neonatal foreskins by immunomagnetic purification and 
cultured as previously described[10]. Briefly, ECs were grown in endothelial cell basal medium (EBM; Lonza, Walkersville, MD, USA) with $20 \%$ FBS, on dishes coated with collagen I at $37^{\circ} \mathrm{C}$ in $5 \% \mathrm{O}_{2}$. LECs were used for experiments in passages 4-8. Human dermal fibroblasts were isolated from human skin biopsies after breast reduction surgeries as previously described[19]. Fibroblasts were grown in DMEM medium (Invitrogen) supplemented with $10 \%$ FCS (HyClone), $100 \mathrm{U} / \mathrm{mL}$ penicillin (Sigma-Aldrich), and 25 $\mu \mathrm{g} / \mathrm{mL}$ gentamicin (Schering), at $37^{\circ} \mathrm{C}$ in $8 \% \mathrm{CO}_{2}$ and atmospheric $\mathrm{O}_{2}$.

In vitro 3D lymphatic vascular construct. A lymphatic vascular construct was generated by culturing human LECs on a connective tissue substitute, which was prepared as described previously, with some modifications[19, 20]. Briefly, dermal fibroblasts were seeded in six well plates at 15,000 cells $/ \mathrm{cm}^{2}$, and cultured for 24 days in complete DMEM supplemented with $50 \mu \mathrm{g} / \mathrm{ml}$ ascorbic acid. Dermal LECs were then seeded on day 24 at a density of 12,000 cells $/ \mathrm{cm}^{2}$ onto the fibroblasts sheets and co-cultures were maintained in a 1:1 mixture of complete DMEM and EGM-2 (Lonza), without adding VEGF, with 50 $\mu \mathrm{g} / \mathrm{ml}$ ascorbic acid. After 7 days of co-culture, on day 31, a stack of three sheets was assembled with two LEC/fibroblast sheets and one fibroblast sheet on top, lifted to the air-liquid interface and cultured for an additional two weeks with ascorbic acid. When inhibitors were used, they were added $4 \mathrm{~h}$ after the seeding of LECs on top of fibroblasts on day 24 , and in every change of media afterwards, three times a week (See scheme of the protocol in Fig. 1A).

Tissue processing and immunostaining. Vascularized constructs were fixed in formaldehyde and perfused with sucrose in PBS as previously described[20]. Each construct was divided in three parts which were (1) formalin-fixed and analyzed as a whole-mount, (2) formalin-fixed and paraffin-embedded (FFPE) for sectioning and (3) embedded in OCT and 
frozen. Immunohistochemistry was done on FFPE sections with rabbit anti-human Prox1 (1:100, Angiobio 11-002), and mouse anti-human CD31 (1:200, Dako M-0823) antibodies, using the catalyzed signal amplification system (CSA Ancillary System, DAKO) according to the manufacturer's protocol. Staining with mouse-anti-human podoplanin antibody (1:50, Dako cl. D2-40, M3619) and rabbit anti-human Ki67 (1:1000, Novocastra NCL-Ki67p) was performed using an automated Leica BOND RX instrument and reagents. Whole-mounts and frozen sections were stained with sheep anti-human CD31 (1:100, R\&D systems AF806), anti-human podoplanin (1:50, Angiobio 11-003), and corresponding secondary antibodies labeled with Alexa-555 or Alexa-633 (Molecular Probes, Eugene, Oregon, USA). Cell nuclei were counterstained with $0.5 \mu \mathrm{g} / \mathrm{ml}$ DAPI (Molecular Probes). Human neonatal foreskins were frozen in OCT compound (Sakura Finetek). Immunofluorescent staining was performed on 6 $\mu \mathrm{m}$ sections as described[21] with mouse anti-human podoplanin (AngioBio), mouse antihuman CD34 (1:100, BD Pharmingen), rabbit anti-human c-Met (1:100, Zymed Laboratories 18-2257) and corresponding secondary antibodies labeled with AlexaFluor-488 or AlexaFluor-594 (Molecular Probes). Cell nuclei were counterstained with $20 \mu \mathrm{g} / \mathrm{ml}$ Hoechst bisbenzimide (Sigma-Aldrich). Tissue sections were examined with a Nikon E600 microscope and images captured with a Nikon DS-Qi1MC camera using NIS Elements software (Nikon). Whole-mounts were examined with a Zeiss LSM 700 single point laser scanning confocal microscope (Zeiss). Image processing and 3D rendering was performed with the Imaris software (Bitplane).

Quantitative analyses of lymphatic vasculature in vitro. FFPE sections of vascular constructs were immunostained with CD31 or podoplanin antibodies and numbers of vessels and lumen areas were determined using NIS Elements software (Nikon). Ki67 was analyzed by manually counting the number of Ki67+ nuclei. Three samples were analyzed per group. 
Total tissue area examined per specimen ranged from 1.6-2.3 $\mathrm{mm}^{2}$. Volume of the lymphatic network and the number of non-connected elements were determined on confocal images of the entire whole-mount sample using Imaris software (Bitplane). Three samples were analyzed per group. Total tissue area examined per specimen was $1.73 \mathrm{~mm}^{2}$.

Transmission Electron Microscopy. Samples were fixed and processed as described previously[22]. Ultra-thin sections stained with uranyl acetate were examined with a transmission electron microscope at $80 \mathrm{kV}$ (JEM-1230, JEOL, Japan).

qRT-PCR. The expression levels of human podoplanin, and c-Met mRNA were quantified by SYBR-Green based Real-Time PCR, using a LightCycler (Roche, Indianapolis, IN, USA). Triplicate reactions containing SYBR Green Jump Start Taq Ready Mix (Sigma, Saint Louis, MO, USA), primers and cDNA were amplified with 40 cycles at $94^{\circ} \mathrm{C}$ for $7 \mathrm{sec}, 58^{\circ} \mathrm{C}$ for 10 sec, and $72^{\circ} \mathrm{C}$ for $10 \mathrm{sec}$. Data were normalized based on $\beta$-actin expression levels. The following primers were used: c-Met: forward 5'-AGTACAAGACTGGGCCTG-3', reverse 5'-AAGTTGATGAACCGGTCC-3';

podoplanin: forward 5'-TGGTGGAATCATCGTTGTGGTTATGC-3', $\quad$ reverse 5'ACAAGTGAACCTCCTGCCTCCTC-3'.

ELISA. Conditioned media were collected from LECs, fibroblasts and co-cultures of LECs and fibroblasts in the 3D lymphatic vascular construct on days 21, 24, 28, 31 and 35 (see outline of the experiment in Fig. 1A). Media were conditioned for the final $48 \mathrm{~h}$ and frozen at $80^{\circ} \mathrm{C}$. HGF and VEGF-C proteins were quantified using corresponding Quantikine ELISA kits (R\&D systems). 
Western blot. For immunoprecipitation cells were lysed in RIPA buffer containing protease and phosphatase inhibitor cocktails (Sigma). $500 \mu \mathrm{g}$ total protein was immunoprecipitated with antibodies to c-Met or VEGFR-3 (Santa Cruz Biotechnology), separated by denaturing SDS-PAGE, transferred to PVDF membrane (Bio Rad Laboratories) and immunoblotted with anti-phospho-Tyrosine antibodies (Santa Cruz Biotechnology). Western blot was performed as described[23]. 15-30 $\mu$ g total protein was separated by denaturing SDS-PAGE, transferred to PVDF membrane and immunoblotted with antibodies to Phospho-ERK1/2, PhosphoSTAT3, Phospho-AKT, STAT3, or AKT (Cell Signaling), and with corresponding secondary antibodies conjugated with HRP (Jackson Immunoresearch). Blots were analyzed with the ECL detection system (Amersham) and densitometry performed with Quantity One software (Bio Rad Laboratories).

Adenoviral transduction and ear whole-mount immunostaining. Ad-HGF, Ad-VEGF-C and Ad-LacZ were provided by Dr. Meenhard Herlyn (The Wistar Institute, Philadelphia, PA, USA)[24]. Viruses were prepared from 293AD embryonic human kidney cells (Cell Biolabs, San Diego, CA, USA), purified using the ViraBind Adenovirus Purification Kit (Cell Biolabs) and titered using the QuickTiter Adenovirus Titer Immunoassay (Cell Biolabs). $5 \times 10^{8}$ pfu of each virus were injected intradermally into the ears of 8-week-old NCR female, athymic nu/nu mice. Mice were sacrificed two weeks after injection. For whole-mount immunofluorescent staining the ears were excised, epidermis removed, and the dermal tissue fixed in acetone/methanol. Tissues were then washed in PBS with 1\% Tween-20 and incubated with anti-mouse LYVE-1 (UBI, Hauppauge, NY, USA) and anti-mouse CD31 (Pharmingen) antibodies, and the corresponding secondary antibodies labeled with AlexaFluor-488 and AlexaFluor-594 (Molecular Probes). Tissue samples were mounted on slides with fluoromount (DakoCytomation, Carpinteria, CA, USA) and examined using a 
Leica TCSSP confocal system (Leica Microsystems, Bannockburn, IL, USA) in conjunction with Velocity High Performance 3D Imaging Software (Improvision Inc., Lexington, MA, USA). All experiments were approved by the IACUC at the Icahn School of Medicine at Mount Sinai.

Proliferation Assay. LECs were plated onto collagen-coated 96-well plates in EBM with $20 \%$ FBS $\left(5 \times 10^{3}\right.$ cells/well, $\left.\mathrm{n}=4\right)$, allowed to adhere for $2 \mathrm{~h}$, starved overnight in EBM with $1 \%$ FBS, and incubated with growth factors for $48 \mathrm{~h}$. BrdU was added during the final $12 \mathrm{~h}$ and fluorescence-based detection was performed using the BrdU cell proliferation kit (EMD Biosciences). Fluorescence was measured at 340/460 nm using a Synergy HT microplate reader (BioTek).

Migration Assay. Transwell inserts (BD Falcon) with $8.0 \mu \mathrm{m}$ pores were coated with collagen and LECs were seeded in EBM with $1 \%$ FBS ( $1 \times 10^{5}$ cells/insert). VEGF-C and HGF were added to the lower compartment and cells were allowed to migrate for $6 \mathrm{~h}$. Inserts were stained with propidium iodide, processed as described[25] and mounted on slides. Migrated cells were examined with a Nikon E-600 fluorescence microscope and quantified with the IPLab software (Scanalytics).

Tube formation in collagen gel. Collagen gel "sandwich" assay was performed as described[10]. Briefly, LECs were seeded on the top of a collagen gel in a 6-well plate $\left(2.5 \times 10^{5}\right.$ cells/well, in EBM $1 \%$ FBS $)$, overlaid with the second layer of collagen, and incubated in EBM 1\% FBS with HGF or VEGF-C for 24h. Phase contrast images were captured using a Nikon TE200 inverted microscope equipped with a SPOT digital camera 
(Diagnostic Instruments). Tube formation was quantified by measuring total tube length per field at 10x magnification using Scion Image software (Scion, Frederick, MD, USA).

Statistical analyses. For quantitative analyses of lymphatic vascular construct in wholemounts, statistical significance was determined using one way ANOVA with a Tukey posttest, for analyses of vasculature in tissue sections, proliferation, migration and tube formation assays statistical significance was determined using two-tailed student's $t$-test. Statistical analyses were made using GraphPad Prism software, version 4.02 for Windows (GraphPad Software, San Diego, CA, USA). All plotted data were expressed as mean \pm SEM.

\section{RESULTS}

\section{D reconstructed lymphatic microvascular network in vitro displays major} morphological and molecular characteristics of the human lymphatic microvasculature.

To generate lymphatic vasculature in vitro, we developed a method for spontaneous formation of a microvascular network in a co-culture of human dermal lymphatic endothelial cells (LECs) and fibroblasts, without any extrinsic scaffold or biomaterial (Fig. 1A). Human adult dermal fibroblasts cultured by the self-assembly approach recreated a physiological environment within three weeks, by secreting and organizing a dense 3D extracellular matrix containing collagens and elastin, with characteristics similar to those observed in vivo[26, 27]. Human LECs added to this connective tissue equivalent spontaneously organized into a 3D lymphatic microvascular network (Fig. 1B-D; Suppl. Fig. 1, 2). Vascular network was dense, branched, and parallel with the plane of the fibroblast sheet, with many vertical connections between the vessels in two planes (Fig. 1D). Lymphatic vessels displayed clear lumens, blind ends, and they were on average $100 \mu \mathrm{m}$ in diameter, recapitulating the morphology of 
lymphatic capillaries in the skin[28] (Fig. 1D, Suppl. Fig. 2). Many sprouts were observed (Fig. 1D, E; Suppl. Fig. 1B), parallel and vertical to the plane, as well as many proliferating LECs (Fig. 1H) (\%Ki67+ LECs in a tissue cross-section: $23 \%, \mathrm{n}=100)$. Vessels were CD31 ${ }^{+}$ and positive for LEC markers podoplanin, LYVE-1 and Prox1[12] (Fig. 1 F-J and Suppl. Fig. 1B, D-I). Immunostaining of the whole network in toto and of tissue sections showed that all CD31+ vessels were also podoplanin+, demonstrating the lymphatic identity of all vessels (Fig. 1F, G and Suppl. Fig. 1 D-I). Vessels consisted of a single layer of LECs, devoid of mural cell coverage, as showed by Prox-1 staining and transmission electron microscopy (TEM) (Fig. $1 \mathrm{~J}-\mathrm{N})$. Vessels were also had poorly developed basement membrane, as shown by TEM and immunostaining for collagen IV (Fig. 1L, and data not shown). TEM further revealed other typical features of the lymphatic capillaries, such as overlapping LEC borders, adherens (button-like) junctions, and anchoring filaments (Fig. 1K-N)[12, 29]. Importantly, the lymphatic network remained stable for the duration of the experiment (three weeks). Thus, we have developed a method for the generation of human lymphatic microvascular network in vitro with cellular, molecular, and ultra-structural characteristics closely resembling native human lymphatic capillaries.

Endogenous production of HGF and VEGF-C in the lymphatic vascular construct. To identify factors which drive spontaneous formation of the lymphatic vasculature in vitro, we screened for the production of secreted factors in the supernatants of $3 \mathrm{D}$ connective tissue constructs with LECs and fibroblasts. Candidates were chosen for analysis based on the likelihood that they mediate a paracrine effect on LECs in our system. We found by RNAseq that LECs expressed very little VEGF-C and no HGF, while they expressed corresponding receptors, VEGFR-3 and c-Met, constitutively and at high levels (data not shown). We did not detect mRNA for PDGF and IGF receptors in LECs by RNAseq (data not shown), although 
their ligands have been reported to mediate lymphangiogenesis[30]. FGF2 and its receptor FGFR1 were found both to be expressed in LECs, suggesting an autocrine mechanism of action, and therefore unlikely to be a key paracrine lymphangiogenesis factor provided by fibroblasts.

We detected significant amounts of VEGF-C and HGF in the co-cultures by ELISA (Fig. 2A). VEGF-C protein was produced by fibroblasts or LECs cultured alone, but VEGF-C levels detected in LEC supernatants were much lower. Comparable amounts of VEGF-C were produced by fibroblasts cultured alone or in co-culture with LECs, suggesting that the major source of VEGF-C production in the construct were fibroblasts. VEGF-C protein levels showed steady, moderate decline within one week of co-culture.

HGF protein levels in the construct were higher than those of VEGF-C. Interestingly, while high levels of HGF protein were detected in co-cultures, level at the limit of detection were observed by ELISA in single cultures of fibroblasts or LECs (Fig. 2A), suggesting that the cross-talk between the LECs and fibroblasts led to the increase in HGF protein levels.

Human dermal LECs abundantly expressed HGF receptor c-Met, as determined by qPCR and Western blotting (Fig. 2B, C). c-Met was constitutively expressed by the quiescent lymphatic endothelium, but not by quiescent blood endothelium (Fig. 2B). c-Met was also expressed by proliferating LECs (Fig. 2C). Microarray analysis of primary microvascular LECs and BECs[10] further confirmed that c-Met was expressed by confluent, growtharrested LECs, but was absent from confluent BECs (GenBank Accession No. HG1747HT1764, identical to J02958, human c-Met complete cds). In normal human skin lymphatic vessels were strongly positive for c-Met, while its expression was absent from most blood vessels (Fig. 2F-I), in agreement with the reports showing that c-Met was highly expressed during angiogenesis, but barely detectable under the normal physiological conditions[31]. 
The c-Met receptor tyrosine kinase on LECs was selectively activated by HGF, as shown by phospho-tyrosine immunoblotting (Fig. 2D) while VEGF-A and VEGF-C did not. Exogenously added VEGF-C selectively phosphorylated VEGFR-3, but HGF had no effect on VEGFR-3 activation (Fig. 2E, and data not shown). Co-precipitation experiments excluded the possibility of a direct interaction between c-Met and VEGFR-3. These data indicate that HGF exerts its effects on human LECs directly through c-Met. Together, the data suggest that the endogenous production of VEGF-C and HGF by dermal fibroblasts drives spontaneous lymphangiogenesis in vitro.

\section{Combined inhibition of c-Met and VEGFR-3 potently diminishes lymphangiogenesis in}

vitro. To investigate the role of HGF/c-Met and VEGF-C/VEGFR-3 axis in the reconstruction of lymphatic vasculature in vitro, we employed a selective inhibitor of c-Met SU11274 and the function-blocking antibody against VEGFR-3. Inhibitors were added from the first day of culturing LECs within the connective tissue equivalent, and incubated for the duration of the experiment (18 days). Analysis of the lymphatic vasculature by immunostaining of the whole constructs and tissue sections for CD31 and podoplanin showed that the inhibition of either VEGFR-3 or c-Met signaling reduced the number of lymphatic vessels and the total volume of the lymphatic network to the comparable extent (Fig. 3A-E). Combined inhibition of VEGFR-3 and c-Met further significantly decreased the number of vessels (median number of vessels per $\mathrm{mm}^{2}$ : control, 118; anti-VEGFR-3, 54; anti-c-Met, 57; or both, 28) (Fig. 3E), as well as the vessel size (median lumen area in $\mu^{2}$ : control, 230; anti-VEGFR-3, 126; anti-cMet, 167; or both, 88) (Fig. 3F). Blocking VEGFR-3 reduced vessel size to a greater extent than blocking c-Met (Fig. 3 A-C F). Combined inhibition of c-Met and VEGFR-3 markedly disrupted organization of a continuous vascular network, and resulted in the formation only of short, truncated, disconnected tubules (Fig. 3 A-C, G). Moreover, we observed many small 
LEC spheroids (Fig. 3A, B), which indicated that the combined treatment also in many cases inhibited tube formation per se. LEC proliferation was also significantly decreased with different treatments (\%Ki67+ LECs in a tissue cross-section: control, 23\%; anti-c-Met, 3\%; anti-VEGFR-3, 3\%; anti-c-Met/VEGFR-3, 3\%; $\mathrm{n}=100$ ). Combined inhibition of c-Met and VEGFR-3 did not decrease LEC proliferation more than inhibition of each receptor alone, suggesting that the additional decrease in network density was due to effects on tubulogenesis in addition to inhibition of proliferation. These data demonstrate a crucial role of combined cMet/VEGFR-3 signaling in the formation of a lymphatic capillary network in vitro.

\section{HGF and VEGF-C have distinct and synergistic roles in lymphangiogenesis in}

vitro. To better characterize and to compare the roles of HGF and VEGF-C during distinct steps of lymphangiogenesis, we next examined their effects on LECs in several in vitro assays. In a BrdU incorporation assay, HGF significantly stimulated proliferation of LECs in a dose-dependent manner $(1-100 \mathrm{ng} / \mathrm{ml})$ and it was slightly more potent than VEGF-C at equal concentrations (Fig. 4A). Both HGF and VEGF-C showed a maximal proliferation effect at $100 \mathrm{ng} / \mathrm{ml}$ (data not shown). Combined treatment with HGF and VEGF-C resulted in a more robust proliferative response compared to each factor alone (Fig. A). This effect was synergistic at low concentrations $(<1 \mathrm{ng} / \mathrm{ml})$, at which neither HGF nor VEGF-C induced proliferation as single factors. In the higher concentration range $(1-100 \mathrm{ng} / \mathrm{ml})$, the combined effects of HGF and VEGF-C on proliferation were near additive (Fig. 4A).

In a Boyden chamber assay, HGF was chemotactic to LECs at 5-100 ng/ml (Fig. 4B). VEGF-C was more potent than HGF in promoting LEC chemotaxis, and it was most effective at higher concentrations (50-100ng/ml). Co-administration of HGF and VEGF-C did not lead to further increase of LEC chemotaxis (data not shown). HGF and VEGF-C also facilitated wound closure in the scratch assay (\% increase over control: HGF 26\%, VEGF-C 60\%, 
HGF+VEGF-C $80 \%$ ). These studies demonstrate that HGF and VEGF-C cooperate in the induction of LEC proliferation, but not chemotaxis, and suggest that the combined effects of HGF and VEGF-C observed in the wound closure assay were due to enhancement of LEC proliferation.

The ability of HGF and VEGF-C to promote formation of lymphatic endothelial tubelike structures was assessed in a 3D collagen gel assay[10]. In the absence of exogenously added growth factors, within $24 \mathrm{~h}$ the majority of LECs failed to generate tubes. Only few cells formed small, non-connecting structures (data not shown). HGF and VEGF-C promoted tubulogenesis only at the high concentration of single growth factors $(100 \mathrm{ng} / \mathrm{ml})$, to a comparable extent (Fig. 4C). Combination of HGF and VEGF-C at 10ng/ml however, showed a synergistic effect (Fig. 4C). In a collagen gel assay tube-like structures formed by LECs were cords which did not contain lumen, and the formation of which was transient; most structures completely regressed by $48 \mathrm{hr}$ (Suppl. Fig. 2 C-E). These structures were very different from the true network of stable vessels formed by LECs in co-cultures with fibroblasts (compare Suppl. Fig. 2A, B with D, E).

Combined effects of HGF and VEGF-C on lymphangiogenesis in vivo. To investigate whether HGF cooperates with VEGF-C in the induction of lymphangiogenesis in vivo, adenoviruses encoding HGF, VEGF-C, or LacZ were injected intradermally into the ears of immunodeficient mice, and the vasculature was analyzed by whole-mount immunostaining for the lymphatic marker LYVE-1. AdVEGF-C induced numerous lymphatic vessel sprouts and extensive lymphatic vascular network formation (Fig. 4E, G). Pronounced sprouting of lymphatics was also observed in the skin of AdHGF transduced ears (Fig. 4 F, H), although HGF gave rise to a less dense lymphatic vasculature as compared to VEGF-C (Fig. 4G, H). Combined adenoviral delivery of HGF and VEGF-C greatly increased lymphatic vascular 
densities when compared with either growth factor alone. A very dense lymphatic network was comprised of both, vastly enlarged lymphatic vessels as well as of numerous thin lymphatic capillaries (Fig. 4I). These results demonstrate that HGF and VEGF-C act in concert to stimulate lymphangiogenesis in vivo.

Synergistic effects of HGF and VEGF-C on LECs are mediated by Erk1/2. To examine the downstream mechanisms by which HGF and VEGF-C cooperate to promote proliferation and tubulogenesis, we assessed activation of MAPK, Akt and STAT3 pathways, because they have been reported as downstream signaling pathways for c-Met and VEGFR-3[30, 32-34]. We first examined Akt, STAT3 and Erk1/2 phosphorylation at $0.25 \mathrm{ng} / \mathrm{ml}$ HGF and VEGF-C, the concentration at which these factors synergistically induced LEC proliferation (Fig. 4G). We found that VEGF-C alone did not phosphorylate Akt (Thr308). HGF alone induced Akt phosphorylation at 10 - 45 min after stimulation, which ceased completely by $90 \mathrm{~min}$. Costimulation with VEGF-C and HGF did not lead to a more robust or prolonged Akt phosphorylation than stimulation with HGF alone (Suppl. Fig. 3A). Furthermore, phosphorylation of STAT3 (Ser727/Tyr705) was not induced by VEGF-C, HGF, nor by the combination of both, within the 5-90 min time-frame (Suppl. Fig. 3A). These data demonstrate that LEC proliferation induced by combined action of HGF and VEGF-C is not mediated by Akt or STAT3 signaling.

In contrast, VEGF-C at $0.25 \mathrm{ng} / \mathrm{ml}$ induced maximal activation of Erk1/2 at $20 \mathrm{~min}$ (phosphorylation Thr202/Tyr204), followed by a decline to near control levels at 45 min (Fig. 5A, B). HGF at $0.25 \mathrm{ng} / \mathrm{ml}$ led to similar levels of Erk1/2 activation at $20 \mathrm{~min}$, but the effect persisted longer, and returned to near control levels by $90 \mathrm{~min}$. Co-stimulation with HGF and VEGF-C at $0.25 \mathrm{ng} / \mathrm{ml}$ led to an earlier onset of Erk1/2 activation and more robust response at 
all time-points examined. Erk1/2 phosphorylation also persisted longer and it remained high even at $90 \mathrm{~min}$, when the effects of single factors were no longer detectable (Fig. 5A, B). Moreover, combined treatment with HGF and VEGF-C at 10ng/ml, a concentration at which synergistic effects on LEC tube formation were observed, also resulted in a more robust Erk1/2 activation compared to HGF or VEGF-C alone (Fig. 5C, D). In contrast, costimulation with HGF and VEGF-C at $10 \mathrm{ng} / \mathrm{ml}$ of each, did not lead to a more robust or prolonged Akt phosphorylation than with each factor alone (Suppl. Fig. 3B). While HGF phosphorylated Akt in the range of concentrations (0.25-100 ng/ml), VEGF-C activated Akt only at high concentration (100ng/ml). Both HGF and VEGF-C phosphorylated STAT3 only at high concentration (100ng/ml) (Suppl. Fig. 3 B, C).

These results demonstrate that the activation of either c-Met or VEGFR-3 on human lymphatic endothelium leads to induction of Erk1/2 signaling, and that the combined stimulation with HGF and VEGF-C increases both, the magnitude and the duration of Erk1/2 activation in LECs. Inhibition of Erk1/2 with the selective inhibitor U0126[35] completely abolished synergistic effects of HGF and VEGF-C on LEC proliferation, and it decreased basal proliferation of LECs in the absence of exogenous growth factors (Fig. 5E). Furthermore, Erk1/2 inhibition with U0126 completely inhibited tube formation induced by HGF and VEGF-C combination treatment (Fig. 5F). U0126 inhibitor was used in the assays at $10 \mu \mathrm{M}$, because this was a minimal concentration required for the complete inhibition of Erk1/2 activation induced by HGF or VEGF-C (Fig. 5G). CellTiter-Blue assay and Trypan blue staining showed no change in LEC viability upon treatment with 1-50 $\mu \mathrm{M}$ U0126 (>95\% viability), ruling out general toxicity of this compound (data not shown). Taken together, these data demonstrate that Erk1/2 signaling is crucial for lymphatic capillary morphogenesis mediated by combined actions of HGF and VEGF-C. 


\section{DISCUSSION}

We describe here a method for generating human lymphatic vasculature in 3D. In this system, human lymphatic endothelial cells, co-cultured with fibroblasts, spontaneously organize into a stable 3D lymphatic capillary network. The method is simple, quantifiable and relevant, as the in vitro-generated lymphatic capillaries exhibit the key cellular, molecular, and ultrastructural characteristics of native, human lymphatic microvasculature. Importantly, the approach is cell-based and it does not require the use of any exogenous biomaterials or growth factors. This model can be used for studies of lymphangiogenesis in vitro, and we corroborate this by demonstrating the important and unique functions of VEGF-C and HGF during distinct steps of lymphangiogenesis. Finally, the construct is directly suitable for transplantation and amenable for incorporation into more complex bioengineered tissues.

Numerous studies have focused on the development of blood vascular constructs in vitro, because blood perfusion is critical for survival of bio-engineered tissues and organ transplants. To date, tissue-engineered blood vessels have been successfully constructed in vitro and used to repair vascular defects among others[36, 37]. In contrast, very few attempts have been made to generate lymphatic vasculature in vitro in a construct which can be adopted for transplantation in humans[14, 15]. In one study human LECs were grown on polyglycolic scaffolds (PGA) which were then successfully transplanted onto mice, however, the LEC formed sheets, not true lymphatic vessels[16]. In another study, pre-vascularized skin grafts were created with human blood and lymphatic endothelial cells embedded in hydrogel[17]. Lymphatics generated within the scaffold anastomosed with the host vasculature when transplanted onto the animals and improved fluid drainage, demonstrating for the first time feasibility of generating functional lymphatics in vitro and the important role of lymphatics in such grafts. Attempts have also been made to regenerate lymphatics in vivo by injecting mesenchymal stem cells, delivering lymphangiogenic factors or transplanting 
lymph nodes[15]. We present here a novel method for generating lymphatic vasculature in a construct suitable for clinical use. The advantage of our method is that (1) it does not involve use of any extrinsic material or growth factors; (2) it generates human lymphatic vessels which extremely well recapitulate all key features of the native human microvasculature; and (3) the system is simple and reproducible.

A lack of relevant in vitro models is an impediment to understanding the mechanisms of lymphangiogenesis. Most studies to date employ 2D cultures of LECs, and 3D assays involve use of scaffolds on which LECs form simple cords or tubes[7]. In contrast, we have developed a lymphangiogenesis assay in which LECs form lymphatic capillaries in 3D which closely resemble native vasculature. Importantly, the lymphatic network remained stable for several weeks, which is in contrast to the majority of assays, in which after the initial phase of endothelial growth and tube formation newly formed tubes rapidly regress. Spontaneous formation of the lymphatic network was driven entirely by combined actions of HGF and VEGF-C endogenously produced by fibroblasts, through c-Met and VEGFR-3 signaling, respectively.

Our results demonstrate different effects of HGF and VEGF-C on distinct steps of lymphangiogenesis in vitro. Compared to VEGF-C, HGF was more effective at inducing LEC proliferation and tube formation, but was less effective at stimulating LEC migration and wound closure. The finding that HGF exerted its most prominent effect on LEC tubulogenesis is in agreement with previous work in other cell systems where it was shown that the HGF/cMet signaling pathway is particularly important for the morphogenesis and organization of the 3D tubular structures[38]. It has been reported previously that HGF participates in lymphangiogenesis[39-41], but conflicting data have been reported regarding its exact role. One study reported expression of c-Met by the LECs in a mouse model of inflammation and by the LECs in vitro[40], and it was proposed that the effects of HGF on LECs were direct. 
The study also suggested that c-Met is a marker of proliferating, activated lymphatic endothelium, in analogy with previous work demonstrating the induction of c-Met expression by angiogenic blood vessels[31]. In contrast, another study reported that HGF induced lymphangiogenesis, but c-Met was not detected on lymphatic vessels in the mouse cornea[39], indicating that HGF acts through an indirect mechanism. Our work clearly demonstrates expression of c-Met by human lymphatic endothelium in vitro and in vivo. This expression pattern was demonstrated by gene profiling, real-time PCR and Western analysis of cultured cells and by the immunostaining of human skin. Surprisingly, we found c-Met to be expressed constitutively and at high levels by quiescent LECs, although in the blood vasculature c-Met expression has been associated with endothelial activation and angiogenesis[31]. In many tissues c-Met is barely detectable under normal physiological conditions, but its expression is dramatically increased in response to injury as it is implicated in repair and regeneration[42]. In human LECs, HGF triggered phosphorylation of c-Met, what resulted in activation of Erk1/2, Akt, and to a lesser extent STAT3 signaling pathways. HGF did not alter the expression levels of VEGFR-3 or VEGFR-2 in LECs, nor did exogenous HGF stimulation result in the trans-phosphorylation of either receptor (data not shown). These results demonstrated that HGF exerts its pro-lymphangiogenic effects independently of VEGF-C, in agreement with the previous study showing that HGF-mediated lymphangiogenesis was not impaired when VEGFR-3 was blocked[40]. Taken together, we demonstrate that c-Met is expressed constitutively by LECs and that HGF promotes lymphangiogenesis directly, by activating c-Met signaling.

An important finding of our study is that the concomitant activation of c-Met and VEGFR-3 resulted in a more dramatic induction of lymphangiogenesis, compared to the activation of each receptor alone. Furthermore, we show that the synergistic effects of HGF and VEGF-C on lymphatic endothelial proliferation and tubulogenesis can be explained by 
activation of MAPK signaling pathway and the elevated activity of Erk1/2. It is interesting to note that at the concentrations where HGF and VEGF-C exerted no biological effect individually, the combination of HGF and VEGF-C induced a robust Erk1/2 activation, resulting in increased LEC proliferation. At higher concentrations, combined stimulation by HGF and VEGF-C further potentiated and sustained Erk1/2 signaling, and thus promoted LEC differentiation and morphogenesis. This is in agreement with previous observations implicating low level MAPK signaling with mitogenic responses, and sustained MAPK signaling with cellular differentiation and morphogenesis[43]. In the context of lymphangiogenesis, one could envision that in the areas distant to the source of growth factors proliferation and mobilization of LECs from the pre-existent lymphatics would occur. As these newly mobilized LECs migrate up the concentration gradient and encounter elevated concentrations of growth factors, they would differentiate to form a more complex lymphatic vascular network.

In conclusion, we have developed a simple and relevant method for the generation of human lymphatic microvascular network in vitro, which is in a construct suitable for testing in vivo. Our model also enables studies of human lymphangiogenesis in vitro in 3D. This model is expected to facilitate studies of lymphangiogenesis and help advance methods for transplantation of lymphatic vasculature in humans, which is important for treating disorders of lymphatics, and for development of new approaches for inhibition of pathological lymphangiogenesis.

\section{ACKNOWLEDGMENTS}

We thank Anita Rogic and Elizabeth Slocum for their invaluable technical assistance, Dr. Sai Krishna Divakar for his help with signaling studies and Drs. Meenhard Herlyn and Steven 
Kazianis for the adenoviral constructs;. This work was supported by the DOD grants BC044819 (M.S.) and BC030921 (B.K.) and CIHR grant SAC92850 (F.A.A.).

\section{AUTHORSHIP CONTRIBUTIONS}

L.G., T.G., F.A.A. and M.S. designed this study. L.G. and T.G. performed tissue engineering experiments. D.A.L. was involved in coordination of experiments and data evaluation. B.K. and S.D. performed experiments - cell culture, molecular biology and in vivo. L.G. and M.S. wrote the manuscript. 


\section{REFERENCES}

1. Wiig, H., et al., Immune cells control skin lymphatic electrolyte homeostasis and blood pressure. J Clin Invest, 2013. 123(7): p. 2803-15.

2. Harvey, N.L., et al., Lymphatic vascular defects promoted by Proxl haploinsufficiency cause adult-onset obesity. Nat Genet, 2005. 37(10): p. 1072-81.

3. Randolph, G.J. and N.E. Miller, Lymphatic transport of high-density lipoproteins and chylomicrons. J Clin Invest, 2014. 124(3): p. 929-35.

4. Card, C.M., S.S. Yu, and M.A. Swartz, Emerging roles of lymphatic endothelium in regulating adaptive immunity. J Clin Invest, 2014. 124(3): p. 943-52.

5. Tewalt, E.F., et al., Lymphatic endothelial cells - key players in regulation of tolerance and immunity. Front Immunol, 2012. 3: p. 305.

6. Szuba, A. and S.G. Rockson, Lymphedema: anatomy, physiology and pathogenesis. Vasc Med, 1997. 2(4): p. 321-6.

7. Bruyere, F. and A. Noel, Lymphangiogenesis: in vitro and in vivo models. FASEB J, 2010. 24(1): p. 8-21.

8. Dixon, J.B., S. Raghunathan, and M.A. Swartz, A Tissue-Engineered Model of the Intestinal Lacteal for Evaluating Lipid Transport by Lymphatics. Biotechnology and Bioengineering, 2009. 103(6): p. 1224-1235.

9. Helm, C.L., A. Zisch, and M.A. Swartz, Engineered blood and lymphatic capillaries in 3-D VEGF-fibrin-collagen matrices with interstitial flow. Biotechnol Bioeng, 2007. 96(1): p. 167-76.

10. Podgrabinska, S., et al., Molecular characterization of lymphatic endothelial cells. Proc Natl Acad Sci U S A, 2002. 99(25): p. 16069-74.

11. Choi, I., et al., 9-cis retinoic acid promotes lymphangiogenesis and enhances lymphatic vessel regeneration: therapeutic implications of 9-cis retinoic acid for secondary lymphedema. Circulation, 2012. 125(7): p. 872-82.

12. Pepper, M.S. and M. Skobe, Lymphatic endothelium: morphological, molecular and functional properties. J Cell Biol, 2003. 163(2): p. 209-13.

13. Bruyere, F., et al., Modeling lymphangiogenesis in a three-dimensional culture system. Nat Methods, 2008. 5(5): p. 431-7.

14. Nipper, M.E. and J.B. Dixon, Engineering the Lymphatic System. Cardiovasc Eng Technol, 2011. 2(4): p. 296-308.

15. Weitman, E., D. Cuzzone, and B.J. Mehrara, Tissue engineering and regeneration of lymphatic structures. Future Oncol, 2013. 9(9): p. 1365-74.

16. Dai, T.T., et al., Reconstruction of lymph vessel by lymphatic endothelial cells combined with polyglycolic acid scaffolds: A pilot study. Journal of Biotechnology, 2010. 150(1): p. 182-189.

17. Marino, D., et al., Bioengineering dermo-epidermal skin grafts with blood and lymphatic capillaries. Sci Transl Med, 2014. 6(221): p. 221 ra14.

18. Persaud, K., et al., Involvement of the VEGF receptor 3 in tubular morphogenesis demonstrated with a human anti-human VEGFR-3 monoclonal antibody that antagonizes receptor activation by VEGF-C. J Cell Sci, 2004. 117(Pt 13): p. 2745-56.

19. Gibot, L., et al., A preexisting microvascular network benefits in vivo revascularization of a microvascularized tissue-engineered skin substitute. Tissue Eng Part A, 2010. 16(10): p. 3199-206.

20. Gibot, L., et al., Development of a tridimensional microvascularized human skin substitute to study melanoma biology. Clin Exp Metastasis, 2013. 30(1): p. 83-90. 
21. Roberts, N., et al., Inhibition of VEGFR-3 activation with the antagonistic antibody more potently suppresses lymph node and distant metastases than inactivation of VEGFR-2. Cancer Res, 2006. 66(5): p. 2650-7.

22. Germain, L., et al., Early basement membrane formation following the grafting of cultured epidermal sheets detached with thermolysin or Dispase. Burns, 1995. 21(3): p. $175-80$.

23. Skobe, M., et al., Induction of tumor lymphangiogenesis by VEGF-C promotes breast cancer metastasis. Nat Med, 2001. 7(2): p. 192-8.

24. Gruss, C.J., et al., Stroma formation and angiogenesis by overexpression of growth factors, cytokines, and proteolytic enzymes in human skin grafted to SCID mice. $\mathrm{J}$ Invest Dermatol, 2003. 120(4): p. 683-92.

25. Das, S., et al., Tumor cell entry into the lymph node is controlled by CCL1 chemokine expressed by lymph node lymphatic sinuses. J Exp Med, 2013. 210(8): p. 1509-28.

26. Pouliot, R., et al., Reconstructed human skin produced in vitro and grafted on athymic mice. Transplantation, 2002. 73(11): p. 1751-7.

27. L'Heureux, N., et al., A completely biological tissue-engineered human blood vessel. FASEB J, 1998. 12(1): p. 47-56.

28. Braverman, I.M., Ultrastructure and organization of the cutaneous microvasculature in normal and pathologic states. J Invest Dermatol, 1989. 93(2 Suppl): p. 2S-9S.

29. Baluk, P., et al., Functionally specialized junctions between endothelial cells of lymphatic vessels. J Exp Med, 2007. 204(10): p. 2349-62.

30. Zheng, W., A. Aspelund, and K. Alitalo, Lymphangiogenic factors, mechanisms, and applications. J Clin Invest, 2014. 124(3): p. 878-87.

31. Ding, S., et al., HGF receptor up-regulation contributes to the angiogenic phenotype of human endothelial cells and promotes angiogenesis in vitro. Blood, 2003. 101(12): p. 4816-22.

32. Makinen, T., et al., Isolated lymphatic endothelial cells transduce growth, survival and migratory signals via the VEGF-C/D receptor VEGFR-3. EMBO J, 2001. 20(17): p. 4762-73.

33. Hilfiker-Kleiner, D., A. Limbourg, and H. Drexler, STAT3-mediated activation of myocardial capillary growth. Trends Cardiovasc Med, 2005. 15(4): p. 152-7.

34. Coso, S., et al., Vascular endothelial growth factor receptor-3 directly interacts with phosphatidylinositol 3-kinase to regulate lymphangiogenesis. PLoS One, 2012. 7(6): p. e39558.

35. Favata, M.F., et al., Identification of a novel inhibitor of mitogen-activated protein kinase kinase. J Biol Chem, 1998. 273(29): p. 18623-32.

36. L'Heureux, N., et al., Human tissue-engineered blood vessels for adult arterial revascularization. Nat Med, 2006. 12(3): p. 361-5.

37. Seifu, D.G., et al., Small-diameter vascular tissue engineering. Nat Rev Cardiol, 2013. 10(7): p. 410-21.

38. Rosario, M. and W. Birchmeier, How to make tubes: signaling by the Met receptor tyrosine kinase. Trends Cell Biol, 2003. 13(6): p. 328-35.

39. Cao, R., et al., Hepatocyte growth factor is a lymphangiogenic factor with an indirect mechanism of action. Blood, 2006. 107(9): p. 3531-6.

40. Kajiya, K., et al., Hepatocyte growth factor promotes lymphatic vessel formation and function. EMBO J, 2005. 24(16): p. 2885-95.

41. Sennino, B., et al., Inhibition of c-Met reduces lymphatic metastasis in RIP-Tag2 transgenic mice. Cancer Res, 2013. 73(12): p. 3692-703.

42. DeLeve, L.D., Liver sinusoidal endothelial cells and liver regeneration. J Clin Invest, 2013. 123(5): p. 1861-6. 
43. Marshall, C.J., Specificity of receptor tyrosine kinase signaling: transient versus sustained extracellular signal-regulated kinase activation. Cell, 1995. 80(2): p. 17985 . 


\section{FIGURE LEGENDS}

Figure 1: Morphological and molecular characteristics of a human 3D reconstructed lymphatic microvascular network in vitro. (A) Schematic outline of the method for in vitro reconstruction of the lymphatic microvascular network within a connective tissue substitute. Image of the construct at the end of experiment is shown on the right. (B, C) Lymphatic vascular network visualized by immunofluorescent staining for CD31 (red), imaged by confocal microscopy (B), or computationally reconstructed using Imaris software (C). (D) Side view of the 3D network shown in $\mathrm{C}$, at higher magnification. Note lymphatic capillary lumens (white arrows), blind-ends (yellow arrows), and sprouts (green arrows). Interconnected networks can be seen in two planes, as indicated with the dotted line. (E) Immunostaining for CD31 and Imaris reconstruction showing numerous filopodia protruding from a lymphatic capillary in vitro. (F, G) Double-immunofluorescent staining for podoplanin (F) and CD31 (G) on the whole reconstructed tissue sample. (H) Immunohistochemical staining for Ki67 (brown) in a tissue cross-section. Cell nuclei are shown in blue. Note Ki67+ LEC in the two vessels shown. (I, J) Immunohistochemistry on serial tissue sections for CD31 (I) and Prox-1 (J). (K-N) TEM images showing typical ultra-structural features of lymphatic capillaries: overlapping endothelial cells $(\mathbf{K}, \mathbf{M}, \mathbf{N})$, button-like adherens junctions (green arrows, $\mathbf{K}, \mathbf{M}, \mathbf{N}$ ), discontinuous basement membrane (red arrow in $\mathbf{L}$ ), anchoring filaments (red arrow in $\mathbf{N}$ ), single layer of LECs, and lack of mural cell coverage $(\mathbf{L}, \mathbf{N})$. Scale bars, $5 \mathrm{~mm}(\mathrm{~A}), 500 \mu \mathrm{m}(\mathrm{B}, \mathrm{C}), 100 \mu \mathrm{m}(\mathrm{D}, \mathrm{F}, \mathrm{G}), 50 \mu \mathrm{m}(\mathrm{H}, \mathrm{I}, \mathrm{J}), 10 \mu \mathrm{m}(\mathrm{E})$, and $500 \mathrm{~nm}$ $(\mathrm{K}-\mathrm{N})$.

Figure 2: Expression of HGF/c-Met and VEGF-C/VEGFR-3 in reconstructed and native dermal lymphatic microvasculature. (A) Production of HGF and VEGF-C protein by 
cultured LECs, fibroblasts, and in 3D co-cultures of LECs with fibroblasts at different days as indicated, determined by ELISA. Error bars indicate SD, n=3. (B) qPCR for human c-Met and podoplanin mRNA in cultured LECs and BECs. (C, D) Western analysis of c-Met protein in LECs at different confluency (C), and upon treatment with HGF, VEGF-C or VEGF-A at 50 ng/ml, as indicated (D). Protein lysates were immunoprecipitated (IP) with c- Met, and immunoblotted (IB) with either phosphotyrosine (p-Tyr) or c-Met antibodies. (E) Western analysis for VEGFR-3 in LECs upon treatment with VEGF-C at $50 \mathrm{ng} / \mathrm{ml}$. Immunoprecipitation with VEGFR-3, immunoblotting with p-Tyr antibodies. (F, G) Immunofluorescent staining of human neonatal foreskin for c-Met (green) and lymphatic marker podoplanin (red). Note selective expression of c-Met by the lymphatic vessels (arrows). (H, I) Immunostaining for c-Met (green, arrows) and CD34 (red, arrowheads) showing lack of c-Met expression on blood vasculature. Cell nuclei are counterstained with Hoechst (blue). Scale bars, $100 \mu \mathrm{m}$.

Figure 3: Effects of HGF/c-Met and VEGF-C/VEGFR-3 inhibition on 3D in vitro lymphangiogenesis. (A, B) Formation of lymphatic microvascular network in the presence of an anti-VEGFR-3 blocking antibody $(2.5 \mu \mathrm{g} / \mathrm{ml})$, HGF inhibitor SU11274 $(1 \mu \mathrm{M})$, or both. Lymphatic vasculature was visualized by immunostaining of the whole construct with a CD31 antibody and reconstructed in $3 \mathrm{D}$ with the Imaris software. $\mathrm{B}$ is a higher magnification of an area shown in A. (C) Cross-sections of the LEC-fibroblast constructs treated with an antiVEGFR-3 antibody, HGF inhibitor or both as indicated, and immunostained for the lymphatic marker podoplanin. (D-G) Quantitative analyses of the lymphatic network volume (D), number of vessels $(\mathbf{E})$, vessel size distribution $(\mathbf{F})$ and connectivity $(\mathbf{G})$ upon treatments as indicated. $* \mathrm{P}<0.05, * * \mathrm{P}<0.01, * * * \mathrm{P}<0.001$. Scale bars, $500 \mu \mathrm{m}(\mathrm{A}), 50 \mu \mathrm{m}(\mathrm{B}), 100 \mu \mathrm{m}(\mathrm{C})$. 
Figure 4: Effects of VEGF-C and HGF on LECs in vitro and on lymphangiogenesis in vivo. (A-C) Comparison of the effects of VEGF-C versus HGF on LEC proliferation, migration and tube formation in vitro: BrdU incorporation (A), migration in a Boyden chamber $(\mathbf{B})$, and tube formation in a collagen gel $(\mathbf{C})$. Data shown are representative of at least three experiments. Error bars indicate SD, $\mathrm{n}=3$. $* \mathrm{P}<0.05$, $* * \mathrm{P}<0.01, * * * \mathrm{P}<0.001$. (D-I) Wholemount staining of mouse ears two weeks after transduction with adenoviruses expressing LacZ control vector, VEGF-C or HGF as indicated. Lymphatic vessels were visualized by immunofluorescent staining with an anti-LYVE-1 antibody. Note increased lymphatic sprouting with VEGF-C (E) or HGF (F) (white arrows). Combination of HGF and VEGF-C markedly increased lymphatic vessel densities (I), when compared to VEGF-C (G) or HGF (E) alone. Scale bars, $100 \mu \mathrm{m}$.

Figure 5: Synergistic effects of HGF and VEGF-C on LECs are mediated via ERK1/2. (A, B) Kinetics of Erk1/2 activation in LECs upon stimulation with VEGF-C, HGF, or both at $0.25 \mathrm{ng} / \mathrm{ml}$ each, concentration at which the combination of HGF and VEGF-C induced LEC proliferation, but single factors did not. LEC protein lysates were immunoblotted with phospho-Erk1/2 and Erk1/2 antibodies (A), and Erk1/2 activation quantified by densitometric analysis (B). (C, D) Kinetics of Erk1/2 activation in LECs upon stimulation with VEGF-C, HGF, or both at $10 \mathrm{ng} / \mathrm{ml}$ each, concentration at which the combination of HGF and VEGF-C induced extensive LEC tube formation, but single factors had only minor effects. LECs protein lysates were immunoblotted with phospho-Erk1/2 and Erk1/2 antibodies (C), and results quantified by densitometric analysis (D). (E, F) Effect of Erk1/2 inhibition on LEC proliferation and tubulogenesis in vitro. LEC proliferation $(\mathbf{E})$ and tube formation in collagen gel (F) upon stimulation with VEGF-C, HGF or both at indicated concentrations, in presence or absence of Erk1/2 inhibitor U0126 at $10 \mu \mathrm{M}$. Note complete inhibition of tube formation in 
the presence of U0126. Data shown are representative of at least three experiments. Error bars indicate $\mathrm{SD}, \mathrm{n}=3$. (G) Inhibition of HGF or VEGF-C-induced activation of Erk1/2 with U0126 at indicated concentrations. 